\title{
Adaptación de la Children's Test Anxiety Scale (CTAS) para su empleo en adolescentes argentinos
}

\author{
Adaptation of the Children's Test Anxiety Scale (CTAS) \\ for its use in Argentine adolescents
}

\author{
Natalia Belén Rivera-Flores ${ }^{1}$, Luis Alberto Furlan * ${ }^{*}$, Gerardo Aníbal Gnavi ${ }^{1}$ \\ 1- Laboratorio de Evaluación Psicológica y Educativa, Facultad de Psicología, \\ Universidad Nacional de Córdoba. Córdoba, Argentina.
}

Introducción

Metodología

Resultados

Discusión

Conclusiones

Referencias

Recibido: 16/03/2019 Revisado: 22/05/2019 Aceptado: 02/06/2019

\section{Resumen}

Se adaptó al español la Children's Test Anxiety Scale (CTAS; Wren \& Benson, 2004) para su empleo en adolescentes argentinos de entre 12 y 17 años. La CTAS es un autoinforme con 30 ítems agrupados en 3 dimensiones: comportamiento fuera de la tarea, pensamientos y reacciones autonómicas. Se realizó la traducción directa de los ítems y el análisis de equivalencia en una muestra bilingüe. Mediante análisis factorial exploratorio $(\mathrm{N}=360$ estudiantes, de ambos sexos, de escuelas secundarias públicas y privadas, de 11 a 13 años, $\mathrm{M}=12.77, \mathrm{DE}=.72$ ) se obtuvieron dos soluciones alternativas para su estructura, una con las tres dimensiones originales y otra de cuatro factores, diferenciando pensamientos de preocupación y autocríticos. Se evaluó el ajuste de ambos modelos con análisis factorial confirmatorio ( $\mathrm{N}=501$ estudiantes, de ambos sexos, de escuelas secundarias públicas y privadas, de 12 a 17 años, $\mathrm{M}$ $=13.98, \mathrm{DE}: .87)$, obteniendo el de cuatro dimensiones los índices más aceptables $\left(\chi^{2} / d f=1.92, \mathrm{CFI}=.90, \mathrm{TLI}=.09\right.$, RMSEA $=0.043, \mathrm{AIC}=410, \mathrm{BIC}=414)$, aunque dos ítems se eliminaron por resultar problemáticos. La consistencia de las escalas fue adecuada $(\alpha=.68-.79)$. Se constataron diferencias de género y correlaciones con el rendimiento académico similares a las informadas con otras escalas de ansiedad frente a los exámenes. Se recomienda el empleo de la adaptación obtenida con algunos recaudos.

Palabras clave: ansiedad ante exámenes; adolescentes; adaptación; análisis factorial, Children's Test Anxiety Scale (CTAS)

\begin{abstract}
The Children's Test Anxiety Scale (CTAS; Wren \& Benson, 2004) was adapted to Spanish for its use in adolescents (12 - 17 years old) from Argentina. CTAS is a self report with 30 items grouped in 3 factors named: task off behaviours, thoughts and autonomic reactions. The items were directly translated into Spanish, and an equivalence analysis in bilingual sample was done. Two alternative solutions to internal structure of the CTAS were obtained through exploratory factor analysis $(\mathrm{N}=360$ students, of both sexes, public and private schools, aged 11 to 13 years, $\mathrm{M}=12.77$, $\mathrm{SD}=.72$ ), one with three original dimensions and another with four factors, distinguishing worry and self - criticism thoughts. Both models fit was assessed through confirmatory factor analysis $(\mathrm{N}=501$ students, of both sexes, public and private schools, aged 12 to 17 years, $\mathrm{M}=13.98 \mathrm{SD}=$ .87), having the four dimension model the most acceptable index $\left(\chi^{2} / d f=1.92, \mathrm{CFI}=.90, \mathrm{TLI}=.09\right.$, RMSEA $=0.043$, $\mathrm{AIC}=410, \mathrm{BIC}=414)$, although two items were eliminated as they were considered problematic. Internal consistency of the scales was adequate $(\alpha=.68-.79)$. Gender differences and correlations with academic performance were corroborated in line with other test anxiety measures. It is recommended to use the adapted version with some precaution.
\end{abstract}

Keywords: test anxiety; teenagers; adaptation; factor analysis; Children's Test Anxiety Scale (CTAS)

* Correspondencia a: Luis Alberto Furlan. Laboratorio de Evaluación Psicológica y Educativa (LEPE), Facultad de Psicología. Universidad Nacional de Córdoba. Enrique Barros esq. Enfermera Gordillo. Ciudad Universitaria, Córdoba (5000), República Argentina. Tel.: +543516196136. E-mail: luis.alberto.furlan@unc.edu.ar

Cómo citar: Rivera-Flores, N. B., Furlan, L. A., \& Gnavi, G. A. (2019). Adaptación de la Children's Test Anxiety Scale (CTAS) para su empleo en adolescentes argentinos. Revista Evaluar, 19(2), 73-89. Recuperado de https://revistas.unc.edu.ar/index.php/revaluar

Nota de autor: Se agradece a la Lic. María José Ferrero, quien colaboró en la recolección de datos y al equipo directivo del The Red Apple School que colaboró para el estudio de equivalencia en muestra bilingüe. 


\section{Introducción}

La ansiedad ante exámenes puede definirse como una tendencia relativamente estable de algunos individuos a responder con elevados niveles de ansiedad ante situaciones en las que sus aptitudes están siendo evaluadas, el aspecto característico es la preocupación por el posible mal desempeño en la tarea y sus consecuencias aversivas para la autoestima, el estatus o la pérdida de algún beneficio esperado (Cassady \& Johnson, 2002; Cizek \& Burg, 2006; Zeidner, 2014).

En un principio, la ansiedad frente a los exámenes (en adelante $\mathrm{AE}$ ) se consideró a los fines teóricos y para su medición una respuesta global y unitaria que incluía manifestaciones cognitivas, somáticas y conductuales (Furlan, 2006). En las décadas de los 60 y 70 del siglo XX hubo dos avances fundamentales en su conceptualización. Por un lado, se distinguió la ansiedad como estado y como rasgo estable de la personalidad (Spielberger, 1966); y por el otro, se diferenciaron dos dimensiones básicas de la experiencia ansiosa, una cognitiva (preocupación) y otra de tipo afectivo - fisiológico (emocionalidad), lo que dio origen al Modelo Dual (Liebert \& Morris, 1967).

Posteriormente, surgieron otros modelos para su medición, debido a que los análisis factoriales realizados sobre los autoinformes de $\mathrm{AE}$ mostraron una estructura interna más compleja que la postulada inicialmente. A nivel cognitivo, en el Reactions to Test (RTT; Sarason, 1984) se distinguieron la preocupación y los pensamientos irrelevantes; y en el German Test Anxiety Inventory, (TAI-G, Hodapp, 1995) la preocupación, la interferencia y la falta de confianza. A nivel afectivo-fisiológico, se diferenciaron los sentimientos de tensión y las reacciones somáticas (Sarason, 1984).

En consecuencia, puede afirmarse que la $\mathrm{AE}$ es un constructo complejo, multidimensional $\mathrm{y}$ dinámico, para el cual se siguen proponiendo nuevas definiciones y procedimientos de medición (Nyroos \& Wiklund-Hörnqvist, 2011) y que por lo general, se ha investigado en poblaciones de jóvenes y adultos, en su mayoría estudiantes universitarios. Sin embargo, la prevalencia de la $\mathrm{AE}$ en niños y adolescentes parece ir en aumento, posiblemente debido al incremento de las pruebas en las escuelas y a las presiones asociadas a este (Wren \& Benson, 2004).

La AE es un constructo relevante para explicar la variabilidad de rendimiento académico, y existe consenso acerca de sus efectos desfavorables sobre el mismo, como lo señalan algunas revisiones ampliamente citadas en este ámbito (Hembree, 1988; Von der Embse \& Hasson, 2012; Zeidner, 1998). Los mecanismos por los cuales se produce dicha influencia comprometen a la atención, la memoria operativa y de largo plazo, el procesamiento de la información y la comprensión lectora, entre otros procesos necesarios para lograr un adecuado desempeño en las evaluaciones (Cassady, 2004; Thomas, Cassady, \& Heller, 2017).

Por otro lado, los estudiantes que presentan elevada AE y solicitan asistencia psicológica por este motivo, presentan en la mayoría de los casos otros síntomas del espectro ansioso, obsesiones, baja autoestima, estado de ánimo deprimido, perfeccionismo y elevada sensibilidad interpersonal (Furlan, 2013). Esta evidencia permite suponer que la presencia de elevada AE constituye un indicador de probables dificultades en el ajuste psicológico del estudiante en otros ámbitos de su vida (Furlan, Ferrero, \& Gallart, 2014).

Un instrumento para evaluar ansiedad ante exámenes en niños o adolescentes debe ser acorde a las características evolutivas de este grupo etario, como así también a las experiencias escolares a las que hayan estado expuestos previamente. En ese sentido, los instrumentos empleados en 
estudiantes universitarios resultan parcialmente adecuados.

El autoinforme más utilizado para niños en edad escolar fue el Test Anxiety Scale for Children (TASC; Sarason, Davidson, Lighthall, Waite, \& Ruebush, 1960). El TASC se administra oralmente y se conceptualiza como una única dimensión, medida por treinta elementos utilizando un formato dicotómico (no/si) de respuesta, la escala se consideró apropiada para los niños en los grados $1^{\text {er }}$ al $6^{\text {to }}$. Sin embargo, este instrumento ha sido cuestionado en las últimas cuatro décadas debido a tres limitaciones: la redacción obsoleta o compleja de los ítems, una definición del dominio desfasada y su dimensionalidad (Wren \& Benson, 2004).

Para superar estas dificultades se construyó la Children's Test Anxiety Scale (CTAS, Wren \& Benson, 2004) y se reunieron evidencias de su validez y fiabilidad para medir la $\mathrm{AE}$ en niños de diversos grupos étnicos. La población seleccionada para su validación $(\mathrm{N}=261)$ fue de estudiantes de los Estados Unidos de América, de $3^{\text {ro }}$ a $6^{\text {to }}$ grado, con edades entre los 8 y 12 años, $53 \%$ mujeres y según su origen, $44 \%$ afroamericanos, 51\% blancos, y 5\% asiáticos. La CTAS fue sometida a un análisis de validez de constructo $\mathrm{y}$, mediante análisis factorial exploratorio, se identificaron tres dimensiones. La primera, denominada pensamiento $(\alpha=.89)$ está conformada por ítems que describen diferentes tipos de cogniciones relacionadas con la actividad en curso o con los resultados esperados. La segunda dimensión, comportamientos fuera de la tarea $(\alpha$ $=.78$ ), posee ítems relacionados con conductas que denotan inquietud o desconexión o dificultades respecto de la tarea. La tercera dimensión, llamada reacciones autonómicas $(\alpha=.85)$ posee ítems relativos a síntomas físicos de tensión emocional y activación del sistema nervioso autónomo. Un análisis confirmatorio aportó evidencias adicionales favorables para esta solución de tres factores (Wren \& Benson, 2004).

La CTAS despertó el interés en los investigadores de países europeos y asiáticos y por eso, se realizaron adaptaciones para su empleo considerando las características de cada cultura. En un estudio reciente, fue traducida al idioma malayo y obtuvo buenos índices de consistencia interna para sus tres subescalas. No se informaron resultados de su estructura interna para esta adaptación (Ali \& Talib, 2015).

También se adaptó al idioma turco (Aydin \& Bulgan, 2017) y se incluyeron estudios de estructura interna mediante análisis factorial confirmatorio ( $\mathrm{N}=1100$ estudiantes, 529 mujeres y 571 varones, de entre 9 y 12 años) que apoyaron el modelo de tres factores de la versión original, se obtuvieron buenos índices de ajuste $\left(\chi^{2} / s d=3.97\right.$, $\mathrm{RMSEA}=.05, \mathrm{SRMR}=.05, \mathrm{CFI}=.97, \mathrm{GFI}=$ $.92, \mathrm{AGFI}=.90)$.

En otra adaptación realizada en Suecia y Finlandia (Nyroos, Korhonen, Linnanmäki, \& Svens-Liavåg, 2012) con una muestra binacional $(\mathrm{N}=172,103$ niñas y 69 niños, de entre 9 y 10 años; se realizó un análisis factorial exploratorio mediante ecuaciones estructurales, que replicó la estructura original de tres factores, aunque logró mejor ajuste para una versión abreviada de 19 ítems que para la original de $30\left(\chi^{2} / s d=1.64\right.$, $\mathrm{RMSEA}=.06, \mathrm{CFI}=.9, \mathrm{TLI}=.90$ )

Otro aspecto considerado a los fines de la validación de la CTAS fue el análisis de las diferencias de género. En los diversos estudios realizados se pudo corroborar lo que plantearon Zeidner (1998), Hembree (1988), Bauermeister, Collazo y Spielberger (1983), Kessler, Sonnega, Bromet, Hughes, \& Nelson (1995), Furlan, Cassady y Pérez (2009) y Heredia, Piemontesi, Furlan y Hodapp (2008) sobre la diferencia en los niveles de ansiedad frente a los exámenes de acuerdo al género. Las mujeres, independientemente 
del entorno cultural y la edad, tienden a informar niveles más altos de $\mathrm{AE}$ que los hombres (Lowe \& Lee, 2008). Similares resultados encontraron Ali y Talib (2015), quienes informaron que las mujeres puntuaron con niveles más altos de $\mathrm{AE}$ en las tres dimensiones, es en la dimensión reacciones autonómicas donde se observó mayor diferencia.

Otras evidencias externas para la validación de las escalas de AE se obtienen mediante el análisis de sus relaciones con algunos indicadores de rendimiento académico (en adelante RA). Al respecto, existen reportes coincidentes de correlaciones negativas y moderadas entre estas variables (Cassady, 2004; Eum \& Rice, 2011; Furlan et al, 2009; Gutiérrez-Calvo, 1996; Hembree, 1998; Von der Embse \& Hasson, 2012; Zeidner, 2014). Sin embargo, cuando se diferencian la contribuciones relativas de cada dimensión de la AE al RA, se observa que algunas dimensiones cognitivas como la interferencia o la falta de confianza, realizan las contribuciones más significativas a la variabilidad del RA, en tanto que la preocupación tiene efectos menos homogéneos, relacionados generalmente con la forma en que esta dimensión se operacionaliza y las reacciones afectivo-fisiológicas o emocionalidad no contribuyen de manera relevante (Piemontesi, Heredia, \& Furlan, 2009).

Por otro lado, la AE constituye un factor de riesgo para el bienestar y desarrollo personal, social y académico de niños y adolescentes (Servera, Llabrés \& Bornás, 1996; La Greca, Siegel, Wallander, \& Walker, 1992), debido a que genera alteraciones específicas en los distintos ámbitos del sujeto, por lo que la ansiedad parece afectar de modo directo a la salud mental en determinadas etapas de la vida, especialmente durante la adolescencia (Axelsson \& Ejlertsson, 2002; Bagley \& Mallick, 2001). En estudios recientes se advierte sobre la importancia de la $\mathrm{AE}$ en relación al bienestar emocional en la infancia y la necesidad de desarrollar acciones que ayuden a prevenirla y mitigarla (Carsley \& Heath, 2019; Segool, Carlson, Goforth, von der Embse, \& Barterian, 2013; Von der Embse, Barterian, \& Segool, 2013).

En ese sentido, y considerando los hallazgos obtenidos en estudiantes universitarios que indican una elevada comorbilidad de la AE y diversos síntomas mentales (Furlan et al, 2014), se podría esperar que la elevada AE en estudiantes secundarios se asocie a la presencia de otros síntomas mentales o relativos al aprendizaje, cuya presencia podría inferirse a partir de la concurrencia a la asistencia psicológica o psicopedagógica.

A pesar de la gran cantidad de literatura internacional, correlatos y tratamientos para la $\mathrm{AE}$, no hay pruebas que evalúen estos aspectos en escolares de nuestro contexto, lo que dificulta conocer el estado de esta problemática a nivel local. En ese sentido, contar con un instrumento adecuado para la población local permitirá realizar con mayor precisión tareas de diagnóstico, detección temprana de estudiantes en situaciones de riesgo de fracaso escolar y con potencial padecimiento subjetivo, estudios de prevalencia, de relaciones entre este constructo y otros que teóricamente se esperaría que estén vinculados, en población adolescente y en intervenciones cuya eficacia se pretenda evaluar.

Para cubrir esta carencia se realizaron cuatro estudios destinados a adaptar la CTAS para su empleo en adolescentes de Argentina. Cabe aclarar que se eligió la población adolescente, que es unos años mayor que la considerada por los autores que construyeron la CTAS, por diversos motivos. El primero es que los exámenes son un dispositivo de evaluación de uso poco frecuente en las escuelas primarias de la Argentina y es en la secundaria, a la cual se accede a partir de los 12 años, cuando comienzan a ser más habituales. Se consideró necesario que la población para la 
cual se adapte la prueba haya tenido experiencias repetidas de exposición ante evaluaciones escritas y orales, ya que la ansiedad frente a los exámenes se define como un rasgo situacional y específico que requiere una tendencia estable de respuesta y surge de ponderar las reacciones emocionales ante una serie de situaciones similares. El segundo motivo para la elección de adolescentes fue la familiaridad con los cuestionarios y las posibilidades de comprender adecuadamente sus consignas y formatos de respuesta. Como se trata de una prueba de autoinforme, es preciso que los estudiantes respondan en forma autónoma y eso requiere una adecuada lectocomprensión, la cual está más garantizada en estudiantes de nivel secundario, en comparación con los de primaria.

El objetivo general que guió los estudios realizados fue elaborar una versión adaptada de la CTAS para su empleo en adolescentes de Argentina. Se establecieron además cuatro objetivos específicos: 1) generar una versión traducida al español de la CTAS y analizar la equivalencia entre esta y su versión original.; 2) reunir evidencias de validez para su estructura interna y evaluar el ajuste de modelos alternativos de medición para la CTAS; y 3) recabar evidencias de validez en base a grupos contrastados, test criterio y de confiabilidad.

Se realizaron estudios para cada objetivo y se formularon las correspondientes hipótesis. 1) Las puntuaciones de las versiones original y traducida se correlacionan en forma positiva y elevada, y las diferencias entre sus medias no son significativas. 2) Los modelos de 3 y 4 factores presentan índices de ajuste adecuados, siendo mejores para el de 4 factores. 3a) Los puntajes de $\mathrm{AE}$ son mayores en mujeres que en varones. $3 b$ ) Los puntajes de AE son mayores en estudiantes con tratamiento psicológico o psicopedagógico, que en los estudiantes sin tratamiento. 3c) La $\mathrm{AE}$ y el rendimiento se correlacionan negativa y moderadamente.

\section{Metodología}

Diseño

Se realizó un estudio de tipo instrumental (Montero \& León, 2002), con cuatro etapas relacionadas con: a) la traducción de los ítems, b) el análisis de la estructura interna y confiabilidad de las subescalas, c) el ajuste de los modelos alternativos de medición $\mathrm{y}$, d) las evidencias test criterio y de grupos contrastados.

\section{Participantes}

En la primera fase, de traducción y análisis de equivalencia, participaron 12 estudiantes bilingües, pertenecientes a una academia privada de inglés, con edades entre los 13 y los 19 años $(\mathrm{M}=16.17 ; \mathrm{DE}=1.64)$. El método de selección fue no probabilístico accidental, cumpliendo con el único requisito de tener idoneidad del idioma inglés para la comprensión del cuestionario.

Para el segundo estudio se empleó una muestra no probabilística accidental, con 360 estudiantes (198 mujeres, 162 varones) de primero y segundo año, con edades entre los 11 y los 13 años $(\mathrm{M}=12.77 ; \mathrm{DE}=.72)$ de escuelas públicas y privadas de Córdoba.

En el tercer y cuarto estudio participaron 501 alumnos (214 mujeres y 287 varones) de 12 a 17 años $(\mathrm{M}=13.98 \mathrm{DE}=.87)$, seleccionados por muestreo no probabilístico accidental de cuatro colegios públicos y privados de la Provincia de Córdoba. 
Instrumentos

Children's Test Anxiety Scale (CTAS; Wren \& Benson, 2004). Posee 30 afirmaciones reunidas en tres escalas: pensamientos (13 ítems, $\alpha=.89$ ), respuestas autonómicas ( 9 ítems, $\alpha=.85$ ) y comportamientos fuera de la tarea (8 ítems, $\alpha=.78$ ), con cuatro opciones de respuesta: Casi nunca, Algunas veces, Mayor parte y Casi siempre, que indican la frecuencia de cada afirmación.

Datos socio académicos. Se elaboró un cuestionario ad hoc, con preguntas relativas al rendimiento obtenido en Lengua y Matemáticas durante el primer y segundo trimestre (promedio autoinformado de calificaciones obtenidas en las evaluaciones de cada materia para cada trimestre). También se indagó sobre la concurrencia a tratamiento psicológico o psicopedagógico durante el año en curso (mediante preguntas específicas con respuestas dicotómicas).

\section{Procedimiento}

En primera instancia se realizó una traducción directa de los 30 ítems del inglés al español. Posteriormente, se verificó su equivalencia administrando la versión original y la traducida a estudiantes bilingües. La administración se realizó en horarios de clases, con previa solicitud de consentimiento a los padres y con una semana de intervalo entre la versión en inglés y en español. Para analizar la equivalencia entre ambos conjuntos de ítems se calculó la prueba $t$ para muestras emparejadas, para el total de los ítems y sus tres subescalas.

Para el primer estudio de estructura interna $(\mathrm{N}=360)$, luego de un análisis preliminar orientado la verificación de los supuestos correspondientes, se realizaron diversos análisis factoriales ex- ploratorios, utilizando el método de componentes principales y rotación promax. La determinación del número de factores se realizó mediante el test de la pendiente (Cattell, 1966) y el análisis paralelo (Horn, 1965). Se utilizó el programa SPSS (IBM Corporation, 2016) versión 24.

Para un nuevo estudio de estructura interna se realizaron análisis factoriales confirmatorios (AFC) basados en las matrices de covarianza, y las soluciones factoriales fueron generadas con la estimación de máxima verosimilitud. Se utilizaron varios índices para evaluar el ajuste entre los modelos y los datos: chi cuadrado sobre grados de libertad $\left(\chi^{2} / d f\right)$, índice de ajuste comparativo (CFI), índice de Tucker-Lewis (TLI) y raíz cuadrada media del error de la aproximación (RMSEA). Los siguientes criterios se utilizaron para evaluar el ajuste del modelo: $\chi^{2} / d f \leq 2.0$ (Hair, Anderson, Tatham, \& Black, 1995); CFI $\geq .90 ;$ GFI $\geq .90 ;$ y RMSEA $\leq .08$ (Browne \& Cudeck, 1993). Para la comparación de múltiples modelos, se utilizaron también el criterio de información de Akaike (AIC) y el criterio de información bayesiano (BIC; mientras más bajos son los valores, mejor es el ajuste). Además, se interpretaron la significación y magnitud de los coeficientes estandarizados de regresión así como la varianza explicada de cada ítem atribuida a su factor latente. Se utilizó el programa Mplus versión 7 (Muthén \& Muthén, 2015) para llevar adelante los análisis estadísticos de acuerdo a los objetivos propuestos.

Para los estudios de evidencias de validez en base a grupos contrastados se empleó la prueba $t$ para muestras no relacionadas y se estimó el tamaño del efecto ( $d$ de Cohen), y para analizar las relaciones con el rendimiento (evidencias test-criterio), se calculó el coeficiente de correlación de Pearson. 


\section{Aspectos éticos}

De acuerdo con los principios éticos vigentes, se informó el objetivo del estudio a los participantes. Al ser menores de edad y debido a que los cuestionarios se administraron en instituciones educativas se solicitó la autorización del personal directivo. Se aseguró la confidencialidad de la información recabada y que los cuestionarios anónimos, así como también el carácter voluntario de su participación. Se comunicó que cualquier sujeto podría abandonar la investigación en el momento que lo deseara. Dado el carácter y tipo de información solicitada a los participantes, no hubo posibilidad de daño en relación con las características de la evaluación. Los instrumentos de evaluación fueron administrados por profesionales idóneos y habilitados para tal fin. Para este estudio se procedió según lo exigen las reglas éticas en vigencia, considerando las pautas propuestas por la American Psychological Association (2017). Para ambos estudios se administró el cuestionario en el horario regular de clases y con autorización previa de los directivos y los padres. La participación fue voluntaria, anónima y los resultados confidenciales.

\section{Resultados}

Equivalencia de las versiones original y traduci$d a$

Se estimaron la prueba $t$ para muestras relacionadas y la correlación de Pearson entre los puntajes totales de las versiones original y traducida de la CTAS. Las medias de los puntajes totales de la versión en inglés y español no difirieron entre sí de forma significativa $(t=-1.51, p$ $=.16)$ y presentaron una correlación elevada $(r=$ $.76, p<.001)$. Resultados similares se obtuvieron respecto de las subescalas de pensamientos $(t=$ - .64, $p=.53, r=.87, p<.001)$, conductas fuera de la tarea $(t=-1.67, p=.12, r=.36, p=.43) \mathrm{y}$ reacciones somáticas $(t=-1.62, p=.13, r=.76$, $p<.001)$. Estos resultados indicaron un nivel de equivalencia aceptable entre ambas versiones.

\section{Análisis factorial exploratorio}

En primera instancia se realizó un análisis para identificar casos atípicos, siendo $\mathrm{z}= \pm 3$ considerados como casos marginales (Tabachnick \& Fidell, 2001), y no se encontraron puntuaciones atípicas. Posteriormente se procedió a verificar el cumplimiento de los supuestos de normalidad; mediante los índices de asimetría y curtosis; y el de multicolinealidad a través de la matriz de correlaciones, resultando ambos satisfactorios.

Los valores de la medida de adecuación muestral Kaiser-Meyer-Olkin $(\mathrm{KMO}=0.88) \mathrm{y}$ la prueba de esfericidad de Bartlett (2822.53, $\mathrm{gl}$ $=435$, sig $=p<.001)$ indicaron la posibilidad de realizar el AFE, el cual se efectuó a través del método de componentes principales y rotación promax. La regla de Kaiser-Guttman indicó la presencia de seis factores, la interpretación del scree test (Cattell, 1966) y un análisis paralelo (Horn, 1965) sugirieron la presencia de cuatro. El cuarto factor surgió de la división de la dimensión cognitiva, que puede diferenciarse en preocupación (más orientada al resultado y sus implicaciones) y pensamientos autocríticos (relacionada con la ejecución). Como el instrumento original estaba conformado por tres factores, se evaluaron dos soluciones alternativas con tres $\mathrm{y}$ cuatro factores.

La solución con tres factores explicó el $36.37 \%$ y la de cuatro factores el $41.64 \%$ total de la varianza, y las escalas obtenidas en ambos casos presentaron valores aceptables de consisten- 
cia interna (ver Tabla 1). Sin embargo, surgieron dificultades para interpretar ambas soluciones por las saturaciones de algunos ítems en factores diferentes a los previstos teóricamente. En la de tres factores, los ítems 5, 6 y 19 que describen cogniciones y el ítem 25 de reacciones autonómicas, saturaron en el factor de conductas no relacionadas a la tarea. El ítem 22 presentó una correlación $<.35$ con el factor correspondiente. En cambio, en la solución de cuatro factores el ítem 22, que es una conducta, correlacionó en forma pequeña (.25) con el factor pensamientos autocríticos y el ítem 25 presentó una correlación de .25 con el factor correspondiente, siendo $<.35$ el punto de corte establecido.

\section{Contraste de modelos alternativos de medición} mediante análisis factorial confirmatorio (AFC)

El estudio se realizó en una nueva muestra $(\mathrm{N}=501)$ de características similares a la empleada en el análisis exploratorio. Se realizó una modificación en la redacción del ítem número 25 Tengo que ir al baño reemplazado por Siento deseos de ir al baño para denominar más claramente una reacción fisiológica y no una conducta.

Los modelos propuestos fueron el de tres factores correlacionados para los 30 ítems de acuerdo a la versión original de la CTAS y el de cuatro factores, con los 30 ítems agrupados según lo encontrado en el análisis factorial exploratorio.

Inicialmente se efectuó una exploración inicial de todos los ítems para evaluar valores perdidos, casos atípicos univariados y multivariados, distribuciones normales univariadas y multivariadas y multicolinealidad. No se encontraron valores perdidos. Luego, los casos atípicos univariados y multivariados fueron identificados calculando los puntajes z estándar para cada variable (puntajes $\mathrm{z}>3.29$ fueron considerados atípicos) y la medida de la distancia de Mahalanobis $(p<$ $.001)$. Los resultados de estos análisis fueron satisfactorios, y se detectaron solo tres casos atípicos multivariados, que no fueron descartados. Los valores de asimetría y curtosis fueron de entre -2 $\mathrm{y}+2$ en todos los ítems, menos uno que superó levemente ese límite en la curtosis. Los valores son considerados aceptables para probar una distribución normal univariada (George \& Mallery, 2010). Al estimar la multicolinealidad de los ítems mediante correlaciones bivariadas de Pearson se obtuvieron valores aceptables para todos los ítems $(r<.90)$.

\section{Estructura interna y consistencia interna del instrumento}

La estimación de los modelos presentó resultados de ajuste por debajo de los valores óptimos. Para el modelo de tres factores los resultados fueron claramente insatisfactorios $\left(\chi^{2} / d f=\right.$ 2.59, $\mathrm{CFI}=.82, \mathrm{TLI}=.81, \mathrm{RMSEA}=.56, \mathrm{AIC}$ $=413, \mathrm{BIC}=417)$. Sin embargo, para el modelo de cuatro factores, el desempeño relativo fue algo mejor con algunos valores adecuados y otros cercanos al límite inferior recomendable $\left(\chi^{2} / d f=\right.$ 2.16, $\mathrm{CFI}=.87, \mathrm{TLI}=.86, \mathrm{RMSEA}=.048, \mathrm{AIC}$ $=411, \mathrm{BIC}=415)$. Posteriormente, se exploraron los índices de modificación y para ambos modelos se especificaron tres relaciones entre pares de errores de medición de ítems que guardaban relación empírica debido a la similitud del contenido. Una nueva estimación de los modelos brindó resultados con evidencias favorables para el de cuatro factores. Específicamente, cada uno de los estadísticos cumplió los criterios estipulados para probar un buen ajuste del modelo (Tabla 3).

Las correlaciones entre los factores fueron medianas tanto en el modelo de $3 \mathrm{~F}(.50$ - .68) como en el de 4F (.48 - .73). Los coeficientes de 
Rivera-Flores, Furlan, \& Gnavi, Evaluar, 2019, 19(2), 73-89

Tabla 1

Análisis factorial exploratorio. Correlaciones ítems-factor de la CTAS para los modelos de 3 y 4 factores.

\begin{tabular}{|c|c|c|c|c|c|c|c|}
\hline \multirow[b]{2}{*}{ Ítem } & \multicolumn{3}{|c|}{ Modelo 3 Factores } & \multicolumn{4}{|c|}{ Modelo 4 Factores } \\
\hline & $\mathbf{P}$ & $\mathbf{R A}$ & CFT & RA & $\mathbf{P P}$ & PA & CFT \\
\hline Me pregunto si aprobaré (1) & .612 & & & & .647 & & \\
\hline Mi corazón late rápido (2) & & .700 & & .714 & & & \\
\hline Miro alrededor del aula (3) & & & .681 & & & & .637 \\
\hline Me siento nervioso (4) & & .576 & & .589 & & & \\
\hline $\begin{array}{l}\text { Creo que voy a obtener una mala califi- } \\
\text { cación (5) }\end{array}$ & & & .385 & & & .826 & \\
\hline $\begin{array}{l}\text { Es difícil para mí recordar las respuestas } \\
\text { (6) }\end{array}$ & & & .525 & & & .609 & \\
\hline Juego con mi lapicera (7) & & & .519 & & & & .536 \\
\hline Siento caliente la cara (8) & & .631 & & .641 & & & \\
\hline Me preocupa fallar (9) & .631 & & & & .592 & & \\
\hline Mi estómago se siente raro (10) & & .537 & & .555 & & & \\
\hline Me preocupa estar haciendo algo mal (11) & .613 & & & & .570 & & \\
\hline Verifico el tiempo (12) & & & .406 & & & & .537 \\
\hline Pienso cual será mi nota (13) & 0.647 & & & & 699 & & \\
\hline Me parece difícil quedarme quieto (14) & & & .439 & & & & .446 \\
\hline $\begin{array}{l}\text { Me pregunto si mis respuestas son correc- } \\
\text { tas (15) }\end{array}$ & .552 & & & & .678 & & \\
\hline Creo que debería haber estudiado más (16) & .396 & & & & & .778 & \\
\hline Me duele la cabeza (17) & & .596 & & .602 & & & \\
\hline Miro a las otras personas (18) & & & .733 & & & & .658 \\
\hline $\begin{array}{l}\text { Creo que la mayoría de mis respuestas son } \\
\text { incorrectas (19) }\end{array}$ & & & .486 & & & .806 & \\
\hline Me hace calor (20) & & .634 & & .644 & & & \\
\hline $\begin{array}{l}\text { Me preocupa lo difícil que es la prueba } \\
\text { (21) }\end{array}$ & .484 & & & & .393 & & \\
\hline Trato de terminar rápido (22) & & & .323 & & & .317 & \\
\hline Me tiembla la mano (23) & & .652 & & .650 & & & \\
\hline Pienso en lo que sucederá si fracaso (24) & .468 & & & & .432 & & \\
\hline Tengo que ir al baño (25) & & & .380 & & & & 276 \\
\hline Muevo los pies (26) & & & .474 & & & & .548 \\
\hline $\begin{array}{l}\text { Pienso en lo mal que lo estoy haciendo } \\
\text { (27) }\end{array}$ & .502 & & & & .441 & & \\
\hline Me siento asustado (28) & & .570 & & .574 & & & \\
\hline Me preocupa lo que dirán mis padres (29) & .559 & & & & .476 & & \\
\hline Me quedo mirando fijamente (30) & & & .446 & & & & .358 \\
\hline $\mathrm{A}$ & .789 & .795 & .766 & .795 & .758 & .750 & .700 \\
\hline $\mathrm{F} 1$ & & & & & & & \\
\hline $\mathrm{F} 2$ & $\mathrm{X}$ & & & & & & \\
\hline F3 & $\mathrm{X}$ & $\mathrm{X}$ & & & & & \\
\hline $\mathrm{F} 4$ & $X$ & $X$ & $X$ & & & & \\
\hline
\end{tabular}


regresión estandarizados entre los factores y los ítems (beta) fueron superiores a .35, a excepción de los ítems 12 (Verifico el tiempo; .27 en $3 \mathrm{~F}$ y .25 en 4F) y 25 (Tengo que [o siento ganas de] ir al baño; 34 en $3 \mathrm{~F}$ y 4F). Por otra parte, la varianza total explicada por los modelos de medición para cada uno de los ítems ( $r$ cuadrado) fue mayor a .10, a excepción del ítem 12 (.07 en 3F y .06 en
4F).

La consistencia interna de las escalas en el Modelo de 3F alcanzó los siguientes valores: pensamientos (13 ítems, $\alpha=.85$ ), reacciones autonómicas (9 ítems, $\alpha=.78$ ) y conductas fuera de la tarea ( 8 ítems, $\alpha=.67 \mathrm{y}$ si se elimina el ítem 12 mejora a $\alpha=.68)$. En el Modelo de 4F:

Tabla 2 Análisis Factorial Confirmatorio. Coeficientes de regresión estandarizados y $r^{2}$ de los ítems de la CTAS para los modelos de 3 y 4 factores.

\begin{tabular}{|c|c|c|c|c|c|c|c|c|c|}
\hline \multirow[b]{2}{*}{ Ítem } & \multicolumn{4}{|c|}{ Modelo 3 Factores } & \multicolumn{5}{|c|}{ Modelo 4 Factores } \\
\hline & $\mathbf{P}$ & RA & CFT & $r^{2}$ & PP & $\mathbf{R A}$ & CFT & PA & $r^{2}$ \\
\hline $\begin{array}{l}\text { Me pregunto si } \\
\text { aprobaré (1) }\end{array}$ & .381 & & & .145 & .423 & & & & .179 \\
\hline $\begin{array}{l}\text { Mi corazón late } \\
\text { rápido }(2)\end{array}$ & & .565 & & .319 & & .571 & & & .326 \\
\hline $\begin{array}{l}\text { Miro alrededor del } \\
\text { aula (3) }\end{array}$ & & & .399 & .159 & & & .409 & & .168 \\
\hline $\begin{array}{l}\text { Me siento nervioso } \\
\text { (4) }\end{array}$ & & .575 & & .331 & & .594 & & & .353 \\
\hline $\begin{array}{l}\text { Creo que voy a } \\
\text { obtener una mala } \\
\text { calificación (5) }\end{array}$ & .399 & & & .301 & & & & .681 & .463 \\
\hline $\begin{array}{l}\text { Es difícil para mí } \\
\text { recordar las respues- } \\
\text { tas (6) }\end{array}$ & .525 & & & .503 & & & & .581 & .337 \\
\hline $\begin{array}{l}\text { Juego con mi lapi- } \\
\text { cera ( } 7)\end{array}$ & & & .450 & .276 & & & .463 & & .214 \\
\hline $\begin{array}{l}\text { Siento caliente la } \\
\text { cara }(8)\end{array}$ & & .490 & & .240 & & .449 & & & .202 \\
\hline $\begin{array}{l}\text { Me preocupa fallar } \\
\text { (9) }\end{array}$ & .612 & & & .374 & .666 & & & & .374 \\
\hline $\begin{array}{l}\text { Mi estómago se } \\
\text { siente raro (10) }\end{array}$ & & .577 & & .333 & & .580 & & & .337 \\
\hline $\begin{array}{l}\text { Me preocupa estar } \\
\text { haciendo algo mal } \\
\text { (11) }\end{array}$ & .664 & & & .441 & .696 & & & & .485 \\
\hline $\begin{array}{l}\text { Verifico el tiempo } \\
\text { (12) }\end{array}$ & & & .268 & .072 & & & .252 & & .064 \\
\hline $\begin{array}{l}\text { Pienso cual será mi } \\
\text { nota (13) }\end{array}$ & .407 & & & .166 & .430 & & & & .185 \\
\hline $\begin{array}{l}\text { Me parece difícil } \\
\text { quedarme quieto } \\
\text { (14) }\end{array}$ & & & .609 & .370 & & & .606 & & .367 \\
\hline
\end{tabular}


Me pregunto si

mis respuestas son $\quad .510$

correctas (15)

Creo que debería

haber estudiado más .545

(16)

Me duele la cabeza

(17)

Miro a las otras

personas (18)

Creo que la mayoría de mis respuestas

son incorrectas (19)

Me hace calor (20)

Me preocupa lo difí-

cil que es la prueba

(21)

Trato de terminar

rápido (22)

Me tiembla la mano

(23)

Pienso en lo que

sucederá si fracaso

(24)

Tengo que ir al baño

(25)

Muevo los pies (26)

Pienso en lo mal

que lo estoy haciendo (27)

Me siento asustado (28)

.612

.325

.395

.628

.570

.468

.219

.483

.623

.388

.498

.248

$356 \quad .126$

.285

.349

.593

.115

.267

.390

.651

.631

.398

.639

.493

.540

.613

.361

.130

.530

.281

.352

.336

.113

.504

.254

Me preocupa lo que dirán mis padres

.482

.232

(29)

Me quedo mirando

fijamente (30)

503

.253

.499

.249

\begin{tabular}{lccccccc}
$\mathrm{F} 1$ & $\mathrm{X}$ & .702 & .613 & $\mathrm{X}$ & .745 & .531 & .531 \\
$\mathrm{~F} 2$ & $\mathrm{X}$ & $\mathrm{X}$ & .540 & $\mathrm{X}$ & $\mathrm{X}$ & .530 & .517 \\
$\mathrm{~F} 3$ & $\mathrm{X}$ & $\mathrm{X}$ & $\mathrm{X}$ & $\mathrm{X}$ & $\mathrm{X}$ & $\mathrm{X}$ & .582 \\
$\mathrm{~F} 4$ & $\mathrm{X}$ & $\mathrm{X}$ & $\mathrm{X}$ & $\mathrm{X}$ & $\mathrm{X}$ & $\mathrm{X}$ & $\mathrm{X}$ \\
\hline
\end{tabular}


pensamientos de preocupación (8 ítems $\alpha=.79$ ), pensamientos autocríticos (5 ítems $\alpha=.79$ ), reacciones autonómicas (9 ítems, $\alpha=.78$ ) y conductas fuera de la tarea (8 ítems, $\alpha=.67 \mathrm{y}$ si se elimina el ítem 12 mejora a $\alpha=.68$ ). En función de los resultados obtenidos se considera adecuado eliminar el ítem 12 y mantener el instrumento con 29 ítems distribuidos en 4 escalas.

Evidencias externas de validez, diferencias de género y test criterio

La prueba $t$ para muestras independientes indicó la existencia de diferencias significativas en las medias de reacciones autonómicas $(p<.001)$, preocupación $(p<.01)$ y AE total $(p<.01)$, que eran mayores en mujeres. Por otro lado, no se observaron diferencias significativas en conductas fuera de la tarea y pensamientos autocríticos.

Respecto de los tratamientos psicológicos, no se constataron diferencias significativas en los niveles de $\mathrm{AE}$ en general, ni en ninguna de sus dimensiones, entre quienes concurren a los mismos $(n=25)$ y quienes no lo hacen $(n=460)$. Los resultados para tratamientos psicopedagógicos fueron similares ( $\mathrm{n}=18$ que asisten).

En cuanto a las evidencias test criterio en relación al rendimiento académico, se obtuvieron correlaciones negativas y moderadas $(r=.30-.33)$ entre la escala de pensamientos autocríticos y las calificaciones parciales en Matemáticas y Lengua. La AE total correlacionó en forma pequeña y negativa $(r=.17-.22)$ con ambos indicadores de rendimiento académico. A diferencia de los pens-

Tabla 3

Ajuste de los 2 modelos de medición de Ansiedad frente a los Exámenes re especificados.

\begin{tabular}{lllllllll}
\hline Modelos & $\chi^{\mathbf{2}}$ & $\boldsymbol{d} \boldsymbol{f}$ & $\chi^{\mathbf{2}} / \boldsymbol{d} \boldsymbol{f}$ & CFI & TLI & RMSEA & AIC & BIC \\
\hline Tres factores & $892.4^{* * * *}$ & 399 & 2.23 & .86 & .85 & .050 & 411 & 415 \\
Cuatro factores & $761.1^{* * * *}$ & 369 & 1.92 & .90 & .89 & .043 & 410 & 414 \\
\hline
\end{tabular}

amientos autocríticos, las otras tres subescalas de AE no presentaron correlaciones significativas.

\section{Discusión}

El proceso de adaptación de la CTAS para su utilización en adolescentes de Argentina permitió obtener una medida de autoinforme que, en términos generales, presenta propiedades satisfactorias de validez y confiabilidad. Respecto de su versión original en inglés, la versión adaptada al castellano posee elevada similitud y a juzgar por los resultados obtenidos en los análisis las correlaciones (elevadas) y diferencias entre grupos (no significativas) su grado de equivalencia puede considerarse aceptable (Hipótesis 1).

En los estudios sobre su estructura interna, la representación el constructo evaluado logra un mejor ajuste con cuatro dimensiones y no con tres como era la propuesta original (Hipótesis 2). Esta subdivisión del componente cognitivo, diferenciando pensamientos de preocupación y pensamientos autocríticos, resulta potencialmente útil a los fines de investigación y aplicados, y coincide con lo propuesto por diversos instrumentos que miden AE en adultos, como el RTT (Sarason, 1984) que distingue preocupación y pensamientos irrelevantes o el TAI-G (Hodapp, 1995) que diferencia preocupación de interferencia.

Estudios realizados localmente con una 
Tabla 4

Evidencias de validez en base a grupos contrastados por género.

\begin{tabular}{lcccc}
\hline & $\begin{array}{c}\text { Mujeres } \\
\mathbf{n = 2 4 1}\end{array}$ & $\begin{array}{c}\text { Varones } \\
\mathbf{n = 2 7 2}\end{array}$ & $\begin{array}{c}\boldsymbol{t} \\
\mathbf{( S i g . )}\end{array}$ & $\boldsymbol{d}$ \\
\hline AE total & 2.32 & 2.16 & 3.41 & .31 \\
& $(.54)$ & $(.47)$ & $* *$ & .29 \\
Preocupación & 2.36 & 2.19 & 3.22 & \\
& $(.59)$ & $(.57)$ & $* *$ & .16 \\
Autocrítica & 2.37 & 2.24 & .36 & .49 \\
& $(.86)$ & $(.70)$ & $\mathrm{n} . \mathrm{s}$ & \\
Reacciones Autonómicas & 2.05 & 1.74 & 5.45 & .18 \\
Conductas fuera de la tarea & $(.66)$ & $(.59)$ & $* *$ & 1.73 \\
\end{tabular}

Nota. $* * * p<.001 . * * p<.01 * p<.05$

versión adaptada del TAI-G (GTAI-AR; Piemontesi, Heredia, \& Furlan, 2012) demostraron que cada dimensión cognitiva del constructo AE se vincula de manera diferente con otras variables, como el rendimiento, el afrontamiento o la autoeficacia para el aprendizaje. La preocupación se vincula de manera positiva con el afrontamiento de aproximación o de orientación al problema, motivando acciones que disminuyan la probabilidad de fracaso, en tanto que la interferencia se asocia con mayor rumiación, autoculpa y menor rendimiento (Piemontesi, Heredia, Furlan, Sánchez-Rosas, \& Martínez, 2012).

En ese sentido, la diferenciación de dos tipos de cogniciones que surgió a partir de los análisis factoriales de la versión adaptada constituye un aporte adicional de valor para comprender las relaciones que cada modalidad de pensamiento establece con otros constructos. Desde el punto de vista temporal, la preocupación estaría evaluando pensamientos anticipatorios que pueden motivar el uso de estrategias auxiliares que prevengan el fracaso (Gutiérrez-Calvo, 1996) mientras que los pensamientos autocríticos, de carácter más retrospectivo, ejercerían interferencia en la realización de las tareas y a nivel motivacional tendrían un efecto menos favorable, lo que provocaría desánimo.

Con respecto a las evidencias externas de validez (Hipótesis 3a) los resultados corroboraron parcialmente las diferencias esperadas en los niveles de AE de acuerdo al sexo, que en el caso de la emocionalidad y la preocupación aparecen más acentuadas en las mujeres (Ali \& Talib, 2015; Lowe \& Lee, 2008). Sin embargo, no se observaron diferencias en la escala de conductas fuera de la tarea y pensamientos autocríticos. Estas discrepancias entre los resultados observados según la dimensión considerada sugieren la necesidad de preservar la conceptualización y medición de la AE como constructo multidimensional, ya que cada uno de sus componentes presenta relaciones específicas con otras variables.

En relación a la sensibilidad de la AE para inferir la presencia de otras condiciones que requieran tratamiento psicológico o psicopedagógico, las diferencias entre grupos no fueron significativas $\mathrm{y}$, en consecuencia, se debe rechazar la hipótesis $3 b$. Probablemente, el reducido tamaño de la muestra que recibía tratamiento (no superó el 10\% del total) sea una limitación para el análisis propuesto y el indicador elegido no sea repre- 
sentativo del fenómeno que se pretendía estimar en forma indirecta.

Finalmente, y tal cual se postuló (Hipótesis $3 \mathrm{c}$ ) la $\mathrm{AE}$ presentó una correlación negativa y moderada con el RA al ser considerada como constructo general (Cassady, 2004; Eum \& Rice, 2011; Furlan et al., 2009; Hembree, 1998; Von der Embse \& Hasson, 2012; Zeidner, 2014). Sin embargo, solo algunas de sus dimensiones presentaron una correlación significativa con el rendimiento y otras no estarían relacionadas con el mismo, como se ha demostrado en estudios realizados con otras medidas multidimensionales de AE (Piemontesi et al., 2012).

\section{Limitaciones}

Los estudios de estructura interna de la versión adaptada de la CTAS se llevaron a cabo en muestras de alumnos con una media de edad superior a la considerada en los estudios originales. Este es un factor que podría suponer limitaciones al momento de comparar los resultados de los análisis realizados en las diferentes versiones de la escala. Los motivos para esta decisión fueron expuestos previamente. Se puede agregar que hay menores probabilidades de sesgos en las respuestas derivados de problemas en la comprensión de las consignas cuando un instrumento construido para niños se administra con adolescentes que si el caso fuera a la inversa. Se espera, de hecho, que la comprensión lectora mejore con la edad y la mayor escolarización y por eso no se considera que esta modificación de la población meta haya podido inducir efectos contraproducentes.

Los niveles de consistencia interna de las escalas obtenidas han sido aceptables mas no plenamente satisfactorios. Mejorarlos supondría reemplazar algunos ítems por otros nuevos que debieran construirse en base a una revisión minuciosa de la definición operacional de cada dimensión. Por otro lado, esta labor excede lo propuesto inicialmente y daría lugar a un instrumento diferente al original, que no permitiría su uso en estudios transculturales.

Otra limitación importante radica en las variables elegidas para reunir evidencias de validez. Se han considerado las diferencias de género e indicadores autoinformados de rendimiento, lo que arrojó resultados similares a los informados con otras medidas de AE.

Sin embargo, no se han evaluado otras variables teóricamente vinculadas con la $\mathrm{AE}$, como la ansiedad general estado - rasgo, ansiedad social, la dimensión neuroticismo o estabilidad emocional de la personalidad, la autoestima o el perfeccionismo. La presencia de sintomatología mental, que frecuentemente se halla en comorbilidad con la AE (Furlan et al., 2014), no se evaluó en forma directa, sino que se estimó en forma indirecta a partir de la concurrencia a servicios de psicología o psicopedagogía, sin que se pudieran constatar diferencias significativas en la $\mathrm{AE}$ entre quienes concurren a los mismos y quienes no lo hacen.

Estudios posteriores podrían considerar la inclusión de algunas de estas variables, como así también otras relativas a los contextos de socialización, como pueden ser las exigencias parentales, el estilo de instrucción del docente o los climas áulicos.

\section{Conclusiones}

La adaptación al español de la CTAS aporta un nuevo instrumento en condiciones para su utilización en las escuelas secundarias de gestión estatal o privada del ámbito local. Su empleo en otras regiones de la Argentina deberá hacerse con 
precaución, especialmente en lo relativo a aspectos característicos del lenguaje de uso frecuente en los adolescentes, de modo de verificar la comprensión de los ítems.

Esta herramienta de evaluación tiene utilidad aplicada con fines de diagnóstico, de tipo clínico individual, como así también para explorar el clima emocional en grupos de estudiantes. Detectar tempranamente los elevados niveles de $\mathrm{AE}$ en los grupos de adolescentes permite implementar estrategias psicoeducativas tendientes a disminuirla, mediante el entrenamiento en estrategias de aprendizaje auto regulado y preparación para rendir, estrategias de regulación emocional y también mediante intervenciones con padres y docentes para modificar factores contextuales asociados con su incremento.

\section{Referencias}

Ali, N. S. M., \& Talib, R. (2015). Test anxiety in school settings: Implication on teachers. Indonesian Journal of Educational Review, 2(2), 67-75. Recuperado de http://pps.unj.ac.id/journal/ijer/index

American Psychological Association. (2017). Ethical Principles of Psychologists and Code of Conduct. Recuperado de http:/www.apa.org/ethics/code/index. aspx

Axelsson, L., \& Ejlertsson, G. (2002). Self-reported health, self-esteem and social support among young unemployed people: A population-based study. International Journal of Social Welfare, 11(2), 111-119. doi: 10.1111/1468-2397.00205

Aydın, U., \& Bulgan, G. (2017). Çocuklarda Sınav Kaygısı Ölçeği'nin Türkçe Uyarlaması. Ilköğretim Online, 16(2), 887-899. doi: 10.17051/ilkonline.2017.304742

Bagley, C., \& Mallick, K. (2001). Normative data and mental health construct validity for the Rosenberg Self-Esteem Scale in British adolescents. International Journal of Adolescence and Youth, 9(2-3),
117-126. doi: 10.1080/02673843.2001.9747871

Bauermeister, J., Collazo, J., \& Spielberger, C. (1983). The construction and validation of the Spanish form of the Test Anxiety Inventory: Inventario de Auto Evaluación sobre Exámenes (IDASE). En C. D. Spielberger \& R. Diaz Guerrero (Eds.). Cross-cultural Anxiety (pp. 67-85). Washington: McGraw-Hill

Browne, M. W., \& Cudeck, R. (1993). Alternative ways of assessing model fit. En: K. A. Bollen \& J. S. Long (Eds.), Testing structural equation models (pp. 136162). Beverly Hills, CA: Sage.

Carsley, D., \& Heath, N. L. (2019). Evaluating the effectiveness of a mindfulness coloring activity for test anxiety in children. The Journal of Educational Research, 112(2), 143-151. doi: 10.1080/00220671.2018.1448749

Cassady, J. C. (2004). The impact of cognitive test anxiety on text comprehension and recall in the absence of external evaluative pressure. Applied Cognitive Psychology, 18(3), 311-325. doi: 10.1002/acp.968

Cassady, J. C., \& Johnson, R. E. (2002). Cognitive test anxiety and academic performance. Contemporary Educational Psychology, 27(2), 270-295. doi: 10.1006/ ceps.2001.1094

Cattell, R. B. (1966). The scree test for the number of factors. Multivariate Behavioral Research, 1(2), 245276. doi: 10.1207/s15327906mbr0102_10

Cizek, G. J., \& Burg, S. S. (2006). Addressing test anxiety in a high-stakes environment: Strategies for classrooms and schools. Thousand Oaks, CA: Corwin.

Eum, K., \& Rice, K. G. (2011). Test anxiety, perfectionism, goal orientation, and academic performance. Anxiety, Stress, \& Coping, 24(2), 167-178. doi: $10.1080 / 10615806.2010 .488723$

Furlan, L. (2006). Ansiedad ante los exámenes. Qué se evalúa y cómo? Evaluar, 6, 32-51. Recuperado de https://revistas.unc.edu.ar/index.php/revaluar

Furlan, L. (2013). Eficacia de una intervención para disminuir la ansiedad frente a los exámenes en estudiantes universitarios argentinos. Revista Colombiana de Psicología, 2(1), 75-89. doi: 10.15446/rcp 
Furlan, L. A., Cassady, J. C., \& Pérez, E. R. (2009). Adapting the Cognitive Test Anxiety Scale for use with Argentinean university students. International Journal of Testing, 9(1), 3-19. doi: 10.1080/15305050902733448

Furlan, L. A., Ferrero, M. J., \& Gallart, G. (2014). Ansiedad frente a los exámenes, procrastinación y síntomas mentales en estudiantes universitarios. Revista Argentina de Ciencias del Comportamiento, 6(3), 31-39. Recuperado de https://revistas.unc.edu.ar/index.php/racc

George, D., \& Mallery, P. (2010). SPSS for Windows step by step: A simple guide and reference, 17.0 Update $\left(10^{\mathrm{a}}\right.$ ed.). Boston, MA: Pearson.

Gutiérrez-Calvo, M. (1996). Ansiedad y deterioro cognitivo: Incidencia en el rendimiento académico. Ansiedad y Estrés, 2(2-3), 173-194. Recuperado de https:// dialnet.unirioja.es

Hair, J. F. J., Anderson, R. E., Tatham, R. L., \& Black, W. C. (1995). Multivariate data analysis (4 ${ }^{\mathrm{a}}$ ed.). Saddle River, NJ: Prentice Hall.

Hembree, R. (1988). Correlates, causes, effects, and treatment of test anxiety. Review of Educational Research, 58(1), 47-77. doi: 10.2307/1170348

Heredia, D., Piemontesi, S., Furlan, L., \& Hodapp, V. (2008). Adaptación del Inventario Alemán de Ansiedad frente a los Exámenes: GTAI-A. Revista Evaluar, 8(1), 46-60. Recuperado de https://revistas.unc. edu.ar/index.php/revaluar

Hodapp, V. (1995). The TAI-G: A multidimensional approach to the assessment of test anxiety. En C. Schwarzer \& M. Zeidner (Eds.), Stress, Anxiety, and Coping in Academic Settings, (pp. 95-130). Francke, Tübingen.

Horn, J. L. (1965). A rationale and test for the number of factors in factor analysis. Psychometrika, 30(2), 179185. doi: $10.1007 /$ bf02289447

IBM Corporation. (2016). IBM SPSS Statistics for Windows, Version 24.0 [software de cómputo]. Armonk, NY: IBM.

Kessler, R. C., Sonnega, A., Bromet, E., Hughes, M., \&
Nelson, C. B. (1995). Posttraumatic stress disorder in the national comorbidity survey. Archives of General Psychiatry, 52, 1048-1060.

La Greca, A. M. E., Siegel, L. J., Wallander, J. L., \& Walker, C. (1992). Stress and coping in child health. Nueva York, NY: Guilford.

Liebert, R. M., \& Morris, L. W. (1967). Cognitive and emotional components of test anxiety: A distinction and some initial data. Psychological Reports, 20(3), 975978. doi: 10.2466/pr0.1967.20.3.975

Lowe, P. A., \& Lee, S. W. (2008). Factor structure of the Test Anxiety Inventory for Children and Adolescents (TAICA) scores across gender among students in elementary and secondary school settings. Journal of Psychoeducational Assessment, 26(3), 231-246. doi: 10.1177/0734282907303773

Montero, I., \& León, O. G. (2002). Clasificación y descripción de las metodologías de investigación en Psicología. International Journal of Clinical and Health Psychology, 2(3), 503-508. Recuperado de http:// www.aepc.es/ijchp/busca.php?coid=Espa\%F1ol

Muthén, L. K., \& Muthén, B. O. (1998-2015). Mplus User’s Guide ( $7^{\mathrm{a}}$ ed.). Los Angeles, CA: Muthén \& Muthén.

Nyroos, M., Korhonen, J., Linnanmäki, K., \& Svens-Liavåg, C. (2012). A cross-national comparison of test anxiety in Swedish and Finnish grade 3 pupils: Measured by the CTAS. Education Inquiry, 3(4), 615636. doi: 10.3402/edui.v3i4.22057

Nyroos, M., \& Wiklund-Hörnqvist, C. (2011). Introducing national test in Swedish primary education: Implications for test anxiety. Electronic Journal of Research in Educational Psychology, 9(25), 995-1022. doi: 10.25115/ejrep.v9i25.1466

Piemontesi, S., Heredia, D., \& Furlan, L. (2009). Correlatos de la ansiedad ante los exámenes: Una aproximación a la teoría de la reducción en la eficiencia. Revista Tesis, 2, 74-86. Recuperado de https://revistas.unc. edu.ar/index.php/tesis

Piemontesi, S., Heredia, D., \& Furlan, L. (2012). Propiedades psicométricas de la versión en español revisada del Inventario Alemán de Ansiedad ante Exámenes 
(GTAI-AR) en estudiantes argentinos. Universitas Psychologica, 11(1), 177-186.

Piemontesi, S., Heredia, D., Furlan, L., Sánchez, J., \& Martínez, M. (2012). Ansiedad ante los exámenes y estilos de afrontamiento ante el estrés académico en estudiantes universitarios. Anales de Psicología, 28(1), 89-96.

Sarason, I. G. (1984). Stress, anxiety, and cognitive interference: Reactions to tests. Journal of Personality and Social Psychology, 46, 929-938.

Sarason, S. B., Davidson, K. S., Lighthall, F. F., Waite, R. R., \& Ruebush, B. K. (1960). Anxiety in elementary school children: A report of research. Hoboken, NJ: John Wiley \& Sons. doi: 10.1037/14349-000

Segool, N. K., Carlson, J. S., Goforth, A. N., von der Embse, N., \& Barterian, J. A. (2013). Heightened test anxiety among young children: Elementary school students' anxious responses to high-stakes testing. Psychology in the Schools, 50(5), 489-499. doi: $10.1002 /$ pits. 21689

Servera, M.; Llabrés, J., \& Bornás, X. (1996). Prevención de la ansiedad en escolares. Ansiedad y Estrés, 2(23), 283-295.

Spielberger, C. D. (1966). Theory and research in anxiety. En C. D. Spielberger (Ed.), Anxiety and Behavior (pp. 3-20). Nueva York, NY: Academic. doi: 10.1016/ b978-1-4832-3131-0.50006-8

Tabachnick, B., \& Fidell, L. (2001). Using multivariate statistics ( $4^{\mathrm{a}}$ ed.). Boston: Allyn and Bacon.

Thomas, C. L., Cassady, J. C., \& Heller, M. L. (2017). The influence of emotional intelligence, cognitive test anxiety, and coping strategies on undergraduate academic performance. Learning and Individual Differences, 55, 40-48. doi: 10.1016/j.lindif.2017.03.001

Von der Embse, N., Barterian, J., \& Segool, N. (2013). Test anxiety interventions for children and adolescents: A systematic review of treatment studies from 20002010. Psychology in the Schools, 50(1), 57-71. doi: $10.1002 /$ pits. 21660

Von der Embse, N., \& Hasson, R. (2012). Test anxiety and high-stakes test performance between school settings:
Implications for educators. Preventing school failure: Alternative Education for Children and Youth, 56(3), 180-187. doi: 10.1080/1045988X.2011.633285

Wren, D. G., \& Benson, J. (2004). Measuring test anxiety in children: Scale development and internal construct validation. Anxiety, Stress, \& Coping, 17(3), 227240. doi: 10.1080/10615800412331292606

Zeidner, M. (1998). Test anxiety: The state of the art. Nueva York, NY: Plenum.

Zeidner, M. (2014). Anxiety in education. En R. Pekrun \& L. Linnenbrink-Garcia (Eds.), International Handbook of Emotions in Education (pp. 265-288). New York, NY: Routledge. doi: 10.4324/9780203148211. $\operatorname{ch} 14$ 